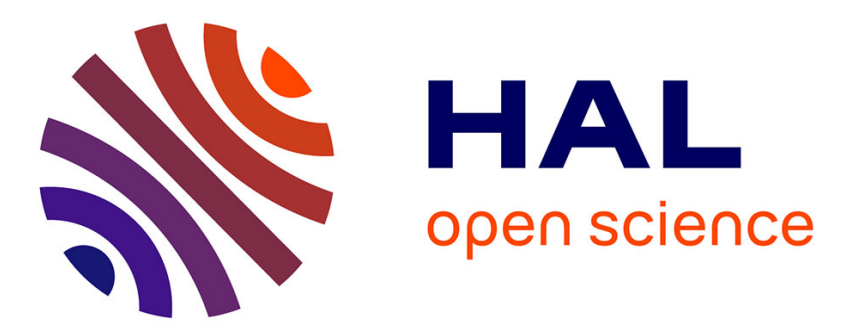

\title{
Résolution de l'ambiguïté sémantique des noms propres par utilisation des croyances sur les connaissances d'autrui - application au prénom
}

Raoul Blin

\section{- To cite this version:}

Raoul Blin. Résolution de l'ambiguïté sémantique des noms propres par utilisation des croyances sur les connaissances d'autrui - application au prénom. Lingvisticae Investigationes, 2017, 40 (2), pp.200 - 227. 10.1075/li.00004.bli . hal-01674582

\author{
HAL Id: hal-01674582 \\ https://hal.science/hal-01674582
}

Submitted on 27 Feb 2018

HAL is a multi-disciplinary open access archive for the deposit and dissemination of scientific research documents, whether they are published or not. The documents may come from teaching and research institutions in France or abroad, or from public or private research centers.
L'archive ouverte pluridisciplinaire HAL, est destinée au dépôt et à la diffusion de documents scientifiques de niveau recherche, publiés ou non, émanant des établissements d'enseignement et de recherche français ou étrangers, des laboratoires publics ou privés. 


\title{
Résolution de l'ambiguiité sémantique des noms propres par utilisation des croyances sur les connaissances d'autrui - application au prénom
}

\author{
Raoul Blin \\ CNRS-CRLAO
}

\section{Introduction}

Un nom propre peut être porté par des centaines de milliers d'individus. Pourtant, dans certaines distributions, ce nom propre ne fait plus référence qu'à un individu unique (polysémie contextuelle (Gosselin, 1996)), non quelconque, et identique pour le locuteur et l'allocutaire. Nous utiliserons le terme «d'univocité» pour désigner la réunion de ces trois conditions. L'univocité est plus contraignante que l'unicité, qui ne pose qu'une contrainte de nombre. Ainsi, dans Je lis un livre. , un pose une contrainte d'unicité, mais pas d'univocité : locuteur et allocutaire peuvent penser à un livre différent, à supposer qu'ils en ont un particulier en tête. Le processus de désambiguïsation mis en œuvre par l'allocutaire pour « retrouver » la référence univoque fait intervenir des données hétérogènes, traditionnellement traitées par des disciplines distinctes. Kleiber (1981) et d'autres linguistes ont évoqué l'influence du contexte sur l'interprétation des noms propres. S'inspirant de l'intelligence artificielle, Renaud (1996), Enjalbert (1996) etc. ont « matérialisé » la notion de contexte en l'identifiant à l'ensemble des connaissances utilisées au moment d'interpréter un énoncé. Blin (2009) a en plus pris en compte la dimension interpersonnelle en suggérant que l'interprétation pouvait jouer sur la distinction entre connaissances propres et connaissances attribuées. Il intègre ainsi la notion de «théorie de l'esprit» de la psychologie à laquelle l'intelligence artificielle s'est aussi intéressée. En traitement automatique du langage, les chercheurs se sont penchés sur les phénomènes de préférence thématique (en cas d'ambiguïté, une interprétation est préférée à une autre si elle est plus proche du thème du discours), et de prépondérance quantitative d'une interprétation (privilégier l'interprétation la plus fréquente). 
L'articulation entre ces données hétérogènes dans le processus d'interprétation et de réduction du sens à une valeur univoque est mal connue et n'a été modélisée que partiellement. Notre objectif est de proposer un modèle formel opératoire du processus, qui intègre l'ensemble de ces données. Comme nous ne pouvons traiter chacune d'elles avec la même attention, nous nous concentrons ici sur le rôle de la théorie de l'esprit dans le processus, tout en indiquant où et comment intégrer les autres données, même si nous ne les décrivons pas de façon aussi poussée. Pour mettre en place le dispositif, nous sommes aussi contraint de réduire la variété des objets linguistiques observés. Nous nous concentrerons sur l'interprétation du prénom. D'une part parce qu'il est très fréquent et répandu. D'autre part parce qu'il existe dans de nombreuses langues et peut servir aux comparaisons entre langues. Enfin, c'est un des mots que l'enfant est amené à entendre et interpréter très tôt. $\mathrm{Ne}$ serait-ce que son propre prénom. Nous proposons ici un modèle pour les adultes mais nous espérons que sa confrontation avec les observations chez les enfants permettra de comprendre le processus de développement de la théorie de l'esprit dans le langage chez l'enfant (Norimatsu et al., 2014).

L'exposé est divisé en trois parties. Tout d'abord, nous délimitons notre problématique linguistique et proposons une représentation lexicale, non quantifiée. Nous décrivons ensuite en détail les connaissances et la façon dont elles sont modélisées pour décrire l'usage de la théorie de l'esprit dans le processus de désambiguïsation du prénom. La section suivante est consacrée au listage des règles d'interprétation du prénom. Nous finissons en intégrant ces règles dans un modèle opératoire formalisé.

\section{Conventions}

Les formules logiques sont écrites dans le langage de la théorie des types (Andrews, 1986). Le langage contient deux types de base, $l$ (individuel) et $o$ (propositionnel). Les types $\alpha, \beta$ et $\gamma \ldots$ représentent des types quelconques, construits à partir des types de base. Nous adoptons la notation de Renaud (1996), avec cependant quelques variantes. Comme en PROLOG, les symboles en minuscules sont des constantes, en majuscule des variables. Pour plus de lisibilité, le typage est implicite, sauf nécessité pour l'exposé. Un sous-terme de la forme $p(X, Y, \ldots)$ se com-

prend $\left(\ldots\left(\left(p_{\alpha \beta \gamma} \ldots X_{\beta}\right) Y_{\gamma}\right) \ldots\right)$. Le point désigne l'application: (A.B) se comprend (AB). Toute chaîne non vide $S$ de caractères est considérée comme une entité et se voit attribuer une constante individuelle notée $S$ et typée $\boldsymbol{~}$. Pour la distinguer des autres constantes, nous conviendrons de lui attribuer l'indice $\sigma$. Hors formules lo-

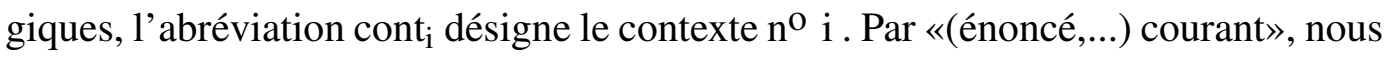




\section{Raoul Blin}

entendons «(énoncé,...) en cours d'analyse». A la manière des identifiants uniques des bases de données, une entité (y compris un événement) se voit attribuer une constante qui lui est propre, une «clef». Deux entités et événements distincts ne peuvent avoir une même clef. Une entité ne peut avoir deux clefs distinctes. Pour simplifier, nous dirons d'une clef affectée à une entité qu'elle la «singularise».

Pour des raisons expliquées plus loin, nous reprenons globalement les analyses sémantiques de Renaud. Le lecteur non familier avec ces analyses peut préalablement consulter le début de la section 7.1 où est présentée une petite grammaire pour un énoncé complet. Le lecteur aura ainsi une vue d'ensemble.

\section{Cadre théorique}

Rappelons très brièvement ce qu'est la théorie de l'esprit. Premack et Woodruff (1978) considèrent qu'un individu a une théorie de l'esprit dès lors qu'il attribue à lui-même et autrui des états mentaux (connaissances, croyances, intentions etc.). Le terme de «théorie» rend compte du fait que l'individu n'a pas une connaissance objective de l'état mental d'autrui, et qu'il reconstitue cet état. Nous adoptons une lecture plus restrictive. Dans le présent article, le terme désigne la représentation que se fait un individu de la cognition (connaissances et raisonnements) d'autrui. Rapporté à la situation de l'interprétation, cela désigne les connaissances et raisonnements que l'allocutaire attribue au locuteur. Une théorie (de l'esprit) est donc un ensemble de connaissances (données et règles d'inférences appliquées à ces données), peu importe que ces connaissances soient objectives ou non. Au même titre que les connaissances propres d'un individu, nous proposons de représenter une théorie de l'esprit sous la forme d'une base de connaissances. Notre tâche est de décrire l'articulation entre la base de connaissances correspondant à une théorie et celle rassemblant les connaissances propres, et de montrer comment le processus de désambiguïsation puise dans ces deux bases.

En cognition et IA, les modèles opératoires qui intègrent la théorie de l'esprit sont implémentés de diverses manières. Sabouret et Belaid (2014) par exemple recourent à des systèmes logiques, tandis que d'autres comme Pynadath et Marsella (2005) utilisent des systèmes procéduraux à base de modèles de Markov. Dans la lignée de ce qui se fait en sémantique linguistique formelle, nous optons pour les systèmes logiques. Ce faisant, nous garantissons la compatibilité entre ces données cognitives et les représentations sémantiques linguistiques, que nous formalisons à l'aide de langages logiques.

Principalement trois modèles logiques sont disponibles. Le modèle DRT (Kamp \& Reyle, 1993) est très utilisé en linguistique pour décrire l'interface sé- 
mantique pragmatique mais n'a pas été spécifiquement adapté pour traiter la théorie de l'esprit. Les modèles BDI («Belief, Desires, Intentions») (Bratman, 1999) utilisés en IA traitent la théorie de l'esprit mais la partie linguistique est peu développée. Notre choix s'est porté sur un troisième modèle, en partant du constat suivant. L'univocité du prénom s'apparente à celle du groupe nominal marqué par le déterminant défini le/la non générique. Les deux font référence à un individu unique. Celui-ci est connu et identique pour le locuteur et l'allocutaire. Si l'allocutaire ne peut établir la valeur référentielle de le $G N$, alors il demande un complément d'informations. Ainsi, dans un discours collaboratif, si le locuteur ordonne Ferme <la> porte. et qu'il n'y a pas de porte susceptible d'être fermée, l'allocutaire demandera Quelle porte ?. De même il demandera Qui est-ce? si on lui ordonne Téléphone à Tulippo. et qu'il ne connaît aucun prénommé Tulippo. Pour traiter ce cas, Renaud (1996) a proposé un dispositif composé d'une grammaire syntactico-sémantique capable d'exploiter des connaissances enregistrées dans des bases de connaissances. Nous proposons de reprendre le même dispositif pour analyser le prénom en contexte.

Le dispositif de Renaud est constitué d'une grammaire de contraintes intégrées associée à des bases de connaissances. Il a été conçu pour décrire l'ensemble de la langue. En plus de la quantification du nom, il a été utilisé pour décrire entre autres la temporalité verbale en français et en coréen, les expressions spatiales en chinois. La grammaire de contraintes intégrées est une grammaire d'unification qui se distingue essentiellement par le langage de description des contraintes. Elle a été formellement présentée dans Renaud (2005). Les représentations sémantiques, formulées dans le langage fonctionnel typé de la théorie des types, contiennent des fonctions qui lancent des calculs sur les bases de connaissances et peuvent en extraire des données et les intégrer aux représentations. Sur le modèle de l'analyse montégovienne, l'analyseur applique les règles de dérivation morphosyntaxiques et sémantiques pour construire la représentation sémantique des énoncés. La dimension pragmatique est incarnée par les bases de connaissances et par les interactions entre ces bases et les représentations sémantiques de la grammaire. Au stade actuel de développement, le dispositif n'intègre pas la distinction entre connaissances propres et connaissances partagées. Notre tâche consiste à l'introduire.

Une particularité du dispositif est de calculer les valeurs référentielles en cours de dérivation, et non pas une fois celle-ci achevée. En cela, il se démarque de la DRT qui effectue les calculs une fois l'analyse achevée. L'interprétation à la volée est psychologiquement plus réaliste. En effet, l'observation naïve des conversations dans la vie quotidienne laisse penser qu'un auditeur n'attend pas la fin d'un énoncé pour en interpréter les composants. Il n'est pas rare de voir un énoncé interrompu 


\section{Raoul Blin}

par un allocutaire qui embraye sur un nom tout juste évoqué (ex.1). Ce n'est possible que si l'allocutaire a interprété le nom avant même l'achèvement de l'énoncé. Même à l'écrit, il est raisonnable de penser qu'un lecteur n'attend pas la fin de la phrase pour interpréter les noms qui y apparaissent.

(1) - Nicolas m'a dit que....

- Ah non! ne me parle plus de lui!

La modélisation logique des raisonnements avec théorie de l'esprit se fait souvent à l'aide de logiques modales et/ou non monotones. Nous nous démarquons en utilisant la logique classique monotone (certes étendue aux ordres supérieurs). Nous adoptons cette logique pour plusieurs raisons. Tout d'abord, la théorie des types a un pouvoir d'expression qui permet de décrire un très grand nombre de phénomènes sémantiques, de la quantification du nom à l'interprétation du temps verbal. Il serait dommage de se priver de cette puissance d'expression. Par ailleurs, la littérature ne démontre pas que les logiques modales et/ou non monotones sont les seules utilisables pour modéliser les phénomènes linguistiques impliquant la théorie de l'esprit. Actuellement, la plupart des travaux impliquant la théorie de l'esprit décrivent le processus de prise de décision (BDI), partant d'une croyance et aboutissant à une intention. La logique classique monotone est réputée inadaptée. Mais notre objet d'observation est très différent. Nous ne sommes donc pas soumis aux mêmes contraintes. Une troisième raison qui nous pousse à en rester à la logique classique monotone est la très grande variété des données sémantiques concernées par la linguistique d'un côté, et la spécialisation des logiques non classiques de l'autre. Les logiques modales sont conçues pour simuler des aspects très spécifiques de la cognition. Les logiques épistémiques et doxastiques ou encore les «description logics» sont conçues pour décrire les manipulations de connaissances, la logique temporelle pour décrire la compréhension du temps, la logique déontique pour la déontologie, etc. A cela il faut ajouter d'autres logiques, destinées à formaliser d'autres aspects de la cognition, comme la logique des défauts. Or le langage naturel couvre tous ces domaines et peut-être plus. Pour simuler le processus d'interprétation, devrat-on combiner toutes ces logiques? Est-ce possible? Faute de pouvoir y répondre, nous préférons en rester, autant que peut se faire, à la logique classique, qui peut être retranscrite dans une majorité de logiques non classiques.

\section{L'objet linguistique étudié}

Afin de prévenir les effets de bords de notre modèle, nous précisons ici les distributions de prénom que nous travaillons. Nous nous limitons au prénom plutôt que de 
travailler sur le nom propre en général car il n'est pas prouvé que du point de vue syntaxique et sémantique tous les noms propres fonctionnent de la même manière. Tout d'abord, les prénoms diffèrent des autres noms propres par le nombre élevé de valeurs référentielles possibles. Par exemple, à l'heure de la rédaction du présent article, des dizaines de milliers d'individus en France se prénomment Nicolas. Il faut ajouter tous ceux qui se sont prénommés ainsi par le passé, et les personnages de fiction. Au contraire, moins d'une cinquantaine de villes dans le monde ${ }^{1}$ portent ou ont porté le nom de Paris. Est-ce lié à ces chiffres ou pas, il est difficile de le dire, mais les propriétés syntaxiques aussi divergent. Ainsi, il est possible de produire des énoncés génériques avec les prénoms alors que c'est peu envisageable avec les noms de villes :

\section{Les Gérard sont des grognons. ${ }^{2}$}

\section{?? Les Paris sont séduisantes.}

Certains noms propres apparaissent systématiquement avec un déterminant défini, comme par exemple les noms de bâtiment : L'Elysée, le Grand Palais, les rivières et fleuves : la Seine. Cette contrainte ne touche pas les prénoms. A cause de ces différences linguistiques, il n'est pas garanti que la procédure d'univocisation du prénom soit valable pour d'autres noms propres. D'où la nécessité de s'en tenir au prénom dans un premier temps. Nous évoquerons toutefois dans la conclusion la possible utilisation du modèle pour traiter d'autres noms propres.

Nous limitons notre étude à l'emploi le plus fréquent du prénom, à savoir son usage sans déterminant ni complément (nom propre «pur» sans aucun sens lexical associé (Jonasson, 1994)), utilisé pour désigner un individu déjà connu. Nous excluons donc plusieurs cas de notre champ de travail. Nous excluons tout d'abord le prénom précédé d'un article (Ex. 2 et 4) et autres distributions avec compléments (voir une liste dans la section 3.2.7 de Jonasson (1994)). Nous excluons aussi la fonction vocative (Lyons, 1978), dans l'apostrophe (Ex. 5).

\section{Le Gérard, il commence à devenir pénible!}

\section{Viens ici Nicolas!}

Enfin, nous excluons les distributions destinées à «baptiser» un nouvel individu (fonction de nomination (Lyons, 1978) ), c'est à dire à attribuer «en toute connaissance de cause» un nom à un individu donné, que ce soit à l'aide de verbes

1. fr.wikipedia.org/w/index.php ?title=Paris_(homonymie). 25/03/2015.

2. Toute ressemblance avec des personnages existants est fortuite. 
de dénomination Mon frère <s'appelle> Gérard ou d'appositions : Gérard, mon frère, ...; Mon frère, Gérard, ... . Par contre, nous prendrons en compte l'usage du prénom lorsqu'il désigne un individu que le locuteur connaît et qu'il croit connu de l'allocutaire. C'est un phénomène fréquent chez les enfants notamment, lorsqu'ils parlent d'une personne qu'ils connaissent sans se rendre compte que l'allocutaire, lui, ne la connaît pas. Dans ce cas, l'intention de l'enfant n'est pas de «baptiser» un individu. Typiquement, l'adulte demandera C'est qui ce $X$ ? ou encore De qui tu parles?.

Pour finir, nous ne prenons pas en compte les prénoms utilisés pour désigner autre chose que des humains. Nous pensons en particulier à ceux utilisés pour désigner des magasins, comme l'enseigne Nicolas. Une étude syntaxique préalable serait nécessaire pour connaître les distributions qui se confondent avec celles des prénoms d'humains, et ainsi mieux appréhender la proximité entre les deux usages.

\section{Représentation sémantique lexicale du prénom}

Notre modèle traite le prénom en trois étapes. Chaque prénom possède une représentation au niveau lexical. C'est l'objet de la présente section. Cette représentation est ensuite «quantifiée» par la grammaire et éventuellement complétée par l'analyseur, lors de la dérivation. A notre connaissance, l'interprétation (logique) du prénom n'a fait l'objet d'aucune étude linguistique spécifique. Nous devons donc partir des travaux sur le nom propre en général. L'interprétation du nom propre a fait l'objet de joutes théoriques entre deux extrêmes. Le courant dominant, représenté par Kripke (1972), défend l'idée d'un nom propre vide de sens ou «désignateur rigide». Cette thèse semble satisfaire la plupart des usages du nom propre. Il s'oppose à la vision d'un nom propre de sens plein, semblable à un nom commun (Searle, 1971; Fredge, 1971). Nous proposons une représentation sémantique lexicale qui dit peu de choses et qui de ce fait correspond plutôt à l'hypothèse de Kripke. Nous partons de Kleiber (1981) que nous paraphrasons: le prénom est «l'abréviation d'un prédicat qui prénomme». L'idée est facilement formalisable en attribuant au nom une représentation basée sur celle du verbe se prénommer. Si ce verbe se représente seprenomme, nous attribuons à l'entrée lexicale Nicolas la représentation cidessous. Avec une telle représentation, nous faisons d'une pierre deux coups: nous adoptons une représentation pour le prénom, et nous établissons de facto un lien 
logique avec le verbe se prénommer. E est l'index davidsonien (Davidson, 2001) ${ }^{3}$ propre à l'événement/état de se prénommer «nicolas $\sigma$ » appliqué à l'individu $X$ :

\section{L1: Nicolas, prenom, $\lambda \mathrm{X}_{l} \exists \mathrm{E}_{l}$ seprenomme $\left(\mathrm{E}\right.$, nicolas $\left._{\sigma}, \mathrm{X}\right)$}

La représentation lexicale ne contient aucune information sur la quantification. La représentation du prénom est typée de la même manière que la représentation du nom commun «standard», à savoir ol comme par exemple porte ${ }_{\mathrm{ol}}$. Grâce à cela, il peut être traité comme un nom commun lorsqu'il est marqué par un déterminant. La quantification du prénom est alors assurée par le déterminant. Cette distribution ne nous concernant pas, nous renvoyons au traitement du nom commun déterminé par le/la de Renaud (1996). Lorsqu'il apparaît seul dans la distribution que nous étudions, il est pris en charge par une règle de réécriture (désormais RR1) qui le quantifie. C'est sur cette règle que nous travaillons, et plus précisément sur sa composante sémantique.

\section{Représenter le contexte}

Les linguistes qui ont travaillé sur le nom propre ont souligné la sensibilité de son interprétation au contexte (voir Jonasson (1994), section 5.2.2.3, pour une description intuitive). Dans la lignée des travaux en intelligence artificielle notamment, et à l'instar de plusieurs autres linguistes (par exemple Renaud ou encore Enjalbert déjà cités en introduction), nous prenons le parti de restreindre la notion «vague» (Kleiber, 1981) de contexte à l'ensemble des connaissances exploitées pour interpréter un énoncé. Ces connaissances sont transcrites logiquement et enregistrées dans des bases de connaissances. Pour comprendre l'articulation entre analyse sémantique (en interprétation) et connaissances, nous revenons en détail sur la transcription de

3. Une telle représentation permet d'adjoindre des données temporelles pour fixer la période de validité du prénom pour l'individu concerné. Une personne peut en effet changer de prénom. Par exemple, la représentation suivante signifie que l'individu singularisé par la clef c se prénomme « Nicolas » d'une date $t_{1}$ à une date $t_{2}$ :

$$
\ldots \text { seprenomme }\left(E, \text { nicolas }_{\sigma}, c\right) \wedge \text { date }(E)=\left[t_{1}, t_{2}\right]
$$

Manipuler l'index nécessite en réalité la représentation plus complète: $\lambda \times \lambda Q \exists E$ seprenomme $(E$, nicolas, $X) \wedge Q$.E. Pour l'exposé, nous nous en tiendrons à la représentation simplifiée.

Cette représentation se distingue de la plus commune qui attribue un symbole de prédicat mono-argumental à chaque nom propre, y compris les prénoms. Par exemple, le fait de se prénommer Nicolas est rendu par : nicolas $(\mathrm{x})$. L'index peut être utilisé pour simuler l'emploi performatif du verbe de prénommage et du prénom. Désormais, tu te prénommes Nicolas. consiste à attribuer à la date d'énonciation (maint) le prénom Nicolas. Voici une suggestion de représentation, qui fait démarrer la période de validité du prénom au moment d'énonciation:

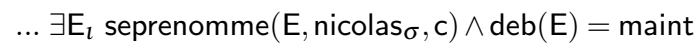




\section{Raoul Blin}

ces connaissances, en particulier sur la dimension interpersonnelle, peu prise en compte dans les descriptions formelles linguistiques. Les connaissances ne constituent pas un bloc monolithique. Nous reprenons la combinaison de deux structures proposée par Blin (2009).

\subsection{Structure 1 des connaissances}

Une première structure, en partie déjà évoquée dans (Renaud, 1996) pour traiter la quantification nominale, est constituée de trois ensembles. Le premier regroupe les connaissances sur le contexte énonciatif: identité du locuteur, de l'allocutaire, et date du discours. Il permet, notamment, de calculer la valeur référentielle des pronoms. Regroupons ces connaissances dans la base de connaissances BCENONCIATIF. Voici les données qui y sont enregistrées pour les énoncés que nous étudierons plus loin (exemple 6, section 6). Chaque énoncé est lui-même considéré comme une entité et singularisé par une clef. Attribuons à l'énoncé la clef $c_{1_{l}}$. Les constantes $\operatorname{luc}_{l}$ et theo ${ }_{l}$ singularisent respectivement le locuteur Luc et l'allocutaire Théo. Nous obtenons:

$$
\text { BCENONCIATIF }=\left\{\operatorname{locuteur}\left(c_{1}\right)=\text { luc }, \text { allocut }\left(c_{1}\right)=\text { theo }, \text { date }\left(c_{1}\right)=\ldots, \ldots\right\}
$$

Cette base ne jouant pas de rôle central pour l'interprétation du prénom, nous n'en discuterons pas en détails. Notons que les connaissances sur le contexte énonciatif sont connues du locuteur et de l'allocutaire et l'allocutaire sait que le locuteur les connaît. Il n'y a donc pas de distinction entre connaissances énonciatives propres et attribuées.

Le second ensemble regroupe les connaissances acquises dans le cotexte de l'énoncé étudié. Nous ne sommes pas en mesure ici de définir le nombre d'énoncés constituant un cotexte et cette question dépasse largement notre propos. Nous l'avons contourné en choisissant d'observer les prénoms dans des énoncés sans cotexte. Dans ce cas, l'ensemble est vide. Comme les connaissances énonciatives, il n'y a pas de distinction entre connaissances propres et attribuées puisque locuteur et allocutaire ont le même cotexte en commun.

Enfin l'allocutaire et le locuteur disposent de «connaissances encyclopédiques». Elles couvrent l'ensemble de l'expérience d'un individu. Les connaissances sont acquises de différentes manières, par le langage ou non. Il existe une relation entre connaissances cotextuelles et connaissances encyclopédiques. Dans un discours ou dialogue, les connaissances énoncées sont dans un premier temps enregistrées comme connaissances cotextuelles. Puis lorsqu'elles n'appartiennent plus au cotexte, elles sont déplacées dans les connaissances encyclopédiques. 


\subsection{Structure 2, dimension interpersonnelle}

La seconde structure, qui rend compte des différences entre locuteur et allocutaire, concerne la base de connaissances encyclopédiques seulement, puisque, comme nous l'avons vu dans la section précédente, le reste des connaissances est partagé. Les connaissances encyclopédiques ne sont pas toutes les mêmes chez le locuteur et l'allocutaire. Deux individus typiques (au sens de la psychologie) sont nécessairement conscients de cette différence. Nous reprenons l'analyse de Blin (2009) qui sépare les connaissances propres de l'allocutaire, ses croyances sur les connaissances du locuteur, et les croyances de l'allocutaire sur les croyances du locuteur sur les connaissances d'autrui.

Pour illustrer la distinction entre les trois ensembles de connaissances et ses effets sur l'interprétation du prénom, nous proposons dans la section 6 suivante d'observer l'interprétation d'un énoncé avec prénom dans plusieurs contextes. Dans la présente section, nous présentons ces contextes et les connaissances dont dispose l'allocutaire dans chacun d'eux.

Contexte 1 : Luc et Théo sont deux amis parisiens, qui étaient au lycée ensemble. Ils sont restés en contact et se revoient régulièrement. Ils ont chacun fait des études supérieures et sont depuis quelques années engagés dans une vie professionnelle. Ils connaissent le personnage historique Nicolas II. Au lycée, les deux ont fait les 400 coups avec un troisième comparse, Nicolas. Ce copain élève désormais des chèvres dans le Tarn. Luc est patron d'une entreprise de services informatiques. Son expert-comptable s'appelle Nicolas Yô. Théo ne le connaît pas et ignore jusqu'à son existence. De son côté, Théo a un lointain cousin prénommé Nicolas dont il n'a jamais parlé à Luc. Enfin, tous les deux savent que le prénom Nicolas est porté par bien d'autres personnes que celles qu'ils connaissent.

Contexte 2 : Le contexte est identique au contexte 1. En plus, Théo connaît Nicolas l'expert-comptable et sait que celui-ci travaille pour Luc. Il sait que Luc ignore qu'il (Théo) connaît ce Nicolas l'expert. Théo peut ainsi affirmer que «Luc ignore que je connais Nicolas, son expert-comptable.».

Contexte 3 : Le contexte est identique au contexte 1. La veille d'envoyer le mail, Théo et Luc ont participé à une soirée, en présence de Nicolas l'expert. Les trois ont discuté ensemble à bâton rompu toute la soirée. Théo et l'expert se sont liés d'amitié. Désormais donc, Théo, Luc et Nicolas l'expert se connaissent et savent qu'ils se connaissent tous les trois. Théo peut affirmer que «Luc sait que je connais Nicolas son expert-comptable.».

Contexte 4 : Identique au contexte 3 . Entre eux, Théo et Luc désignent usuellement leur copain d'enfance à l'aide du diminutif Nico. 
Puisque notre intérêt porte sur le processus d'interprétation, nous nous concentrons sur les connaissances exploitées par l'allocutaire Théo. Nous détaillerons ces connaissances pour le contexte 1. Nous donnerons ensuite les connaissances dans les autres contextes, mais sans les justifier en détails. Enonçons intuitivement les connaissances qui n'impliquent pas de croyances sur les connaissances d'autrui. Nous parlerons de «connaissances propres». Dans le contexte 1, l'allocutaire sait que (p1) un individu s'est appelé Nicolas (II) et (p2) qu'il est mort. L'allocutaire connaît aussi la règle générale que $(\mathrm{p} 3)$ les morts ne téléphonent pas. Il connaît par ailleurs un individu qui est son copain et (p4) qui s'appelle Nicolas. Il sait qu'un autre individu est son cousin et que (p5) il s'appelle Nicolas. Il sait enfin que (p6) bien qu'il n'en connaisse pas d'autres en personne, «Nicolas II, le copain et le cousin ne sont pas les seuls individus au monde à se prénommer Nicolas.». Donnonsnous une fonction $\mathrm{CP}(\mathrm{A}, \mathrm{Cont})$ qui renvoie l'ensemble des connaissances propres (transcrites logiquement) d'un individu A dans le contexte Cont. Les représentations sont skolémisées de sorte à les débarrasser des quantificateurs, à la manière de PROLOG. nicll, copain et expert singularisent respectivement Nicolas II, le copain et l'expert. Nous ne représentons que les connaissances dont l'usage est non trivial ou important pour l'exposé. Ainsi, nous ne représentons pas les relations «copain» et «cousin», inutiles pour l'interprétation. Les représentations sont simplifiées et ne mentionnent pas, notamment, la datation.

$\mathrm{CP}\left(\right.$ allocutaire, cont $\left._{1}\right)=\{$

(p1) seprenomme $\left(e_{2}\right.$, nicolas $_{\sigma}$, nicll $)$,

(p2) $\operatorname{mort}\left(e_{2}\right.$, nicll),

(p3) $\neg \operatorname{mort}\left(E_{3}, X\right) \vee \neg$ telephone $\left(E_{4},\{X\}\right) \vee$ date $\left(E_{4}\right)<\operatorname{date}\left(E_{3}\right)$,

(p4) seprenomme $\left(e_{5}\right.$, nicolas $_{\sigma}$, copain $)$,

(p5) seprenomme $\left(e_{6}\right.$, nicolas $_{\sigma}$, cousin $)$,

(p6) seprenomme $\left(\mathrm{e}_{7}\right.$, nicolas $\left._{\sigma}, \mathrm{c}_{8}\right) \wedge \mathrm{c}_{8} \notin\{$ copain, nicll, cousin $\}$

\}

L'allocutaire peut aussi reconstituer les connaissances du locuteur. Pour reprendre la terminologie de la psychologie, on parlera de «théorie » de l'allocutaire sur les connaissances du locuteur. Nous traitons une théorie sur des connaissances ni plus ni moins que comme un ensemble de connaissances, distinct de celui des connaissances propres. Donnons-nous une fonction $\mathrm{TC}(\mathrm{A}, \mathrm{B}, \mathrm{Cont})$ qui renvoie la théorie de l'individu A sur les connaissances de l'individu B dans le contexte Cont. Pour abréger, nous désignerons par TC l'ensemble renvoyé par la fonction lorsque A est l'allocutaire et B le locuteur pour le contexte courant. Du fait de leur passé commun, l'allocutaire Théo attribue ses propres connaissances au locuteur, à l'exclusion 
de Nicolas le cousin. Pour signifier que l'allocutaire estime que le locuteur connaît une même entité que lui, nous attribuons à cette entité la même clef dans les deux bases de connaissances, CP et TC. L'allocutaire ne peut dire si le locuteur connaît l'expert ni quelles propriétés le locuteur attribue à l'expert puisque l'allocutaire luimême ne connaît pas l'expert. Nous postulons plus généralement que (Règle 1) un individu A ne peut attribuer à autrui la connaissance d'une entité B non quelconque si A ne connaît pas B. Voyons maintenant comment traiter des entités dont l'allocutaire connaît l'existence, dont il sait que le locuteur connaît l'existence, mais parmi lesquelles il ne connaît aucune en particulier: «Je (=allocutaire) sais qu'il existe d'autres personnes prénommés Nicolas, mais je n'en connais aucune en particulier. Le locuteur sait aussi que d'autres personnes existent mais je ne saurais dire si il en connaît une en particulier.». Pour rendre compte de cette situation nous insérons p6 aussi dans TC mais avec de nouvelles constantes de skolem, distinctes des constantes de $\mathrm{p} 6$ dans $\mathrm{CP}$.

$\mathrm{TC}\left(\right.$ allocutaire, locuteur, cont $\left._{1}\right)=\{$

(p1) seprenomme $\left(e_{2}\right.$, nicolas $_{\sigma}$, nicll $)$,

(p2) $\operatorname{mort}\left(e_{2}\right.$, nicll),

(p3) $\neg \operatorname{mort}\left(E_{9}, X\right) \vee \neg$ telephone $\left(E_{10},\{X\}\right) \vee$ date $\left(E_{10}\right)<$ date $\left(E_{9}\right)$,

(p4) seprenomme $\left(e_{5}\right.$, nicolas $_{\sigma}$, copain $)$,

$\left(\mathrm{p6}^{\prime}\right)$ seprenomme $\left(\mathrm{e}_{11}\right.$, nicolas $\left._{\sigma}, \mathrm{c}_{12}\right) \wedge \mathrm{c}_{12} \notin\{$ copain, nicll $\}$

\}

Comme nous l'avons déjà mentionné, notre travail nous amène sur le terrain de la théorie de l'esprit en psychologie, où la notion de fausse croyance ${ }^{4}$ est incontournable. Même si elle ne joue aucun rôle pour l'interprétation du prénom, nous devons la situer dans notre dispositif si nous voulons que le modèle serve aussi en psychologie. Nous postulons qu'attribuer une fausse croyance à autrui consiste à considérer qu'autrui tient pour vraie une connaissance que l'on tient soi-même pour fausse. Supposons un contexte 5 basé sur le contexte 1, où en plus l'allocutaire attribue au locuteur la fausse croyance de l'existence du Père Noël («Le locuteur croit que le Père Noël existe»). Pour rendre compte d'une fausse croyance, nous la tenons pour vraie dans la théorie sur autrui (TC) et fausse dans les connaissances propres (CP):

$\mathrm{CP}\left(\right.$ allocut, cont $\left.t_{5}\right)=\mathrm{CP}\left(\right.$ allocut, cont $\left._{1}\right) \cup\{\neg \exists \mathrm{X}$ perenoel $(\mathrm{X})\}$ $\mathrm{TC}\left(\right.$ allocut, loc, $\left.\mathrm{cont}_{5}\right)=\mathrm{TC}\left(\right.$ allocut, loc, $\left.\operatorname{cont}_{1}\right) \cup\{\exists \mathrm{X} \operatorname{perenoel}(\mathrm{X})\}$

4. Introduite par Dennett (1978), elle a été popularisée par le test dit « de fausse croyance » (Wimmer $\&$ Perner, 1983). C'est aujourd'hui un concept clef en psychologie expérimentale pour observer la capacité à manipuler la théorie de l'esprit. 
Blin (2009) posait l'existence d'une relation d'inclusion entre les ensembles de connaissances : TC $\subseteq$ CP. Cependant l'étude n'intégrait pas les fausses croyances. Notre représentation des fausses croyances est incompatible avec cette relation d'inclusion. En effet, si p est une fausse croyance, alors (1) $\neg p_{o} \in C P$ et (2) $p_{o} \in$ TC. Si la relation d'inclusion était maintenue, nous déduirions de (2) que $\mathrm{p}_{\mathrm{o}} \in \mathrm{CP}$, ce qui rendrait CP logiquement inconsistante et anéantirait notre modélisation des fausses croyances. Il est donc préférable de ne pas reprendre cette inclusion.

Un troisième ensemble joue un rôle crucial pour interpréter le prénom, celui des croyances de l'allocutaire sur les croyances du locuteur sur les connaissances de l'allocutaire. L'allocutaire est capable de reconstituer les connaissances que le locuteur a sur lui, l'allocutaire: «Le locuteur sait/croit que je connais ...», ou, pour reprendre le terme de théorie : «J'ai une théorie sur la théorie du locuteur sur mes connaissances.». Donnons-nous une fonction très générale $\operatorname{TTC}(\mathrm{A}, \mathrm{B}, \mathrm{C}, \mathrm{Cont})$ qui renvoie la théorie de l'individu A sur la théorie de l'individu B sur les connaissances de l'individu $\mathrm{C}$ dans le contexte Cont, et tel que $\mathrm{A} \neq \mathrm{B}$. Pour simplifier, TTC désigne la fonction lorsque $\mathrm{A}=\mathrm{C}=$ allocutaire, $\mathrm{B}=$ locuteur dans le contexte courant. Dans le contexte 1, l'allocutaire peut sans difficulté imaginer que pour le locuteur, le copain est une « connaissance commune ». De même, tout individu ayant leur niveau d'étude a entendu parler de Nicolas II. L'allocutaire s'imagine bien que le locuteur tient aussi ce personnage historique pour une connaissance commune. Le contexte énonciatif est commun aux deux. L'allocutaire ne connaissant pas Nicolas l'expert, il ne peut pas se prononcer sur ce qu'en pense le locuteur, application de la règle 1. Par application de cette même règle, l'allocutaire sait que le locuteur ne connaît pas le cousin, donc que le locuteur ne peut pas se prononcer à propos des connaissances de l'allocutaire sur ce cousin. Le cousin est donc absent de TTC.

TTC(allocutaire, loc, allocutaire, cont $\left._{1}\right)=\{$

(p1) seprenomme $\left(e_{2}\right.$, nicolas $_{\sigma}$, nicll $)$,

(p2) $\operatorname{mort}\left(\mathrm{e}_{2}\right.$, nicll),

(p3) $\neg \operatorname{mort}\left(E_{13}, X\right) \vee \neg$ telephone $\left(E_{14},\{X\}\right) \vee$ date $\left(E_{14}\right)<$ date $\left(E_{13}\right)$,

(p4) seprenomme $\left(e_{5}\right.$, nicolas $_{\sigma}$, copain $)$,

$\left(\mathrm{p6}^{\prime \prime}\right)$ seprenomme $\left(\mathrm{e}_{15}\right.$, nicolas $\left._{\sigma}, \mathrm{c}_{16}\right) \wedge \mathrm{c}_{16} \notin\{$ copain, nicll $\}$

\}

Nous proposons maintenant une synthèse de l'état des connaissances dans les autres contextes. Les bases de connaissances du contexte 2 sont identiques à celles du contexte 1 à ceci près qu'il faut ajouter la connaissance de l'expert (p7) expertcomptable(expert) et de son prénom (p8) seprenomme $\left(e_{17}\right.$, nicolas $_{\sigma}$, expert) dans les connaissances propres de l'allocutaire ( «Je connais l'expert.») et dans TC 
( «Je sais que le locuteur connaît cet expert.»), mais pas dans TTC ( «Le locuteur ne sait pas que je connais cet expert.»).

$\mathrm{CP}($ allocut, cont 2$)=\mathrm{CP}\left(\right.$ allocut, cont $\left._{1}\right) \cup\{\mathrm{p} 7, \mathrm{p} 8\}$

$\mathrm{TC}\left(\right.$ allocut, loc, cont $\left._{2}\right)=\mathrm{TC}\left(\right.$ allocut, loc, cont $\left._{1}\right) \cup\{\mathrm{p} 7, \mathrm{p} 8\}$

TTC(allocut, loc, allocut, cont 2$)=$ TTC(allocut, loc, allocut, cont 1 )

Les connaissances du contexte 3 valent celles du contexte 2 , à cette différence près que l'allocutaire peut affirmer «Le locuteur sait que je connais l'expert.». En conséquence,

$\mathrm{CP}\left(\right.$ allocut, $\left.\mathrm{cont}_{3}\right)=\mathrm{CP}\left(\right.$ allocut, cont $\left._{2}\right)$

TC (allocut, loc, cont $\left._{3}\right)=\mathrm{TC}\left(\right.$ allocut, loc, $\left.\mathrm{cont}_{2}\right)$

TTC(allocut, loc, allocut, cont 3$)=$ TTC(allocut, loc, allocut, cont 2$) \cup\{$ p7,p8 $\}$

Le contexte 4 est identique au 3 à ceci près qu'il comporte l'information sur le surnom du copain. Le surnommage est représenté sur le modèle du prénommage : p9 $=$ sesurnommer $\left(e_{18}\right.$, nico $_{\sigma}$, copain $)$. Les constantes sont identiques dans les trois bases.

$\mathrm{CP}\left(\right.$ allocut, cont $\left._{4}\right)=\mathrm{CP}\left(\right.$ allocut, $\left.\mathrm{cont}_{3}\right) \cup\{\mathrm{p} 9\}$

TC(allocut, loc, cont $\left._{4}\right)=$ TC (allocut, loc, cont $\left._{3}\right) \cup\{\mathrm{p} 9\}$

TTC (allocut, loc, allocut, cont $\left._{4}\right)=$ TTC(allocut, loc, allocut, cont 3$) \cup\{$ p9 $\}$

\subsection{Manipulation des connaissances}

Les bases de connaissances se prêtent à diverses manipulations. Pour interpréter le prénom, nous recourrons à «l'extraction» d'entités. Nous reprenons pour cela la fonction srch_info de Renaud (1996) qui extrait une constante à partir d'un terme. Nous la modifions marginalement pour lui donner un caractère générique. srch_info2 $(\mathrm{o} \alpha) \mathrm{o}(\mathrm{o} \alpha) \cdot$ base $_{\circ} . \mathrm{t}_{\mathrm{o} \alpha}$ renvoie l'ensemble des symboles $\mathrm{t}_{\alpha}^{\prime}$ tels que: base $_{\mathrm{o}} \vdash \mathrm{t}_{\mathrm{o} \alpha} \cdot \mathrm{t}_{\alpha}^{\prime}$.

Par exemple, l'extraction des clefs des individus prénommés Nicolas à partir de la base TTC produit le résultat ci-dessous. ttc $\mathrm{i}_{\mathrm{i}}$ vaut TTC(allocutaire, locuteur,allocutaire,cont $\mathrm{i}_{\mathrm{i}}$. rsem $_{\text {nicolas }}$ est la représentation sémantique de l'entrée lexicale Nicolas (voir L1 dans la section 4). Nous voyons que telle que définie, srch_info2 extrait aussi des constantes qui ne sont pas présentes dans les autres bases (CP et TC). C'est le cas par exemple de $c_{16}$.

$$
\text { srch_info2.ttc } 1 . \text { rsem }_{\text {nicolas }}=\left\{\text { copain, nicll, } \mathrm{c}_{16}\right\}
$$


14 Raoul Blin

$$
\begin{aligned}
& \text { srch_info2.ttc } 2 . \text { rsem }_{\text {nicolas }}=\left\{\text { copain, nicll, } c_{16}\right\} \\
& \text { srch_info2.ttc } 3 . \text { rsem }_{\text {nicolas }}=\left\{\text { copain, nicll, expert, } \mathrm{c}_{16}\right\} \\
& \text { srch_info2.ttc } 4 . \text { rsem }_{\text {nicolas }}=\left\{\text { copain, nicll, expert, } c_{16}\right\}
\end{aligned}
$$

\section{Interprétation du prénom en contexte, observations et analyses}

La présente section liste les règles ${ }^{5}$ d'interprétations du prénom en contexte. Ces règles «transforment» la représentation sémantique lexicale. Elles seront implémentées dans le modèle. Nous illustrons l'exposé à l'aide d'un dialogue simple que nous plongeons dans nos quatre contextes. Il s'agit d'un mail envoyé par Luc (locuteur) à Théo (allocutaire). Nous considérons ce mail comme le premier tour de parole dans un dialogue car le locuteur s'adresse à un individu spécifique dont il attend une réponse. L'allocutaire en a pleinement conscience. L'occurrence étudiée de $\mathrm{Ni}$ colas se trouve dans le premier énoncé. La salutation qui précède n'apporte aucune information pertinente pour l'exposé. Il n'y a donc pas de cotexte.

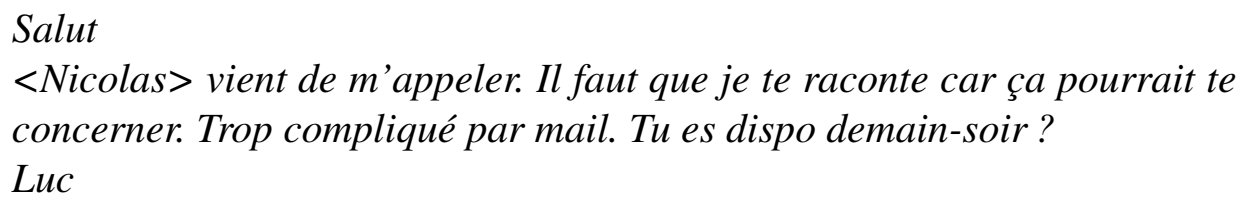
concerner. Trop compliqué par mail. Tu es dispo demain-soir?

Luc

\subsection{Unicité de l'individu auquel il est fait référence}

Comme cela a été évoqué de multiple fois plus haut, (Règle 2) en contexte le prénom désigne un individu unique. Cette contrainte est rendue par un quantificateur existentiel à valeur unique. Voici la première version de la règle de réécriture RR1 (dans les règles sémantiques, rsem $\mathrm{i}$ désigne la représentation sémantique du i-ème composant du syntagme).

$$
\begin{aligned}
& (\mathrm{RR} 1, \mathrm{v} 1) \mathrm{GN} \leftarrow \text { prenom, } \\
& \lambda \mathrm{P} \exists_{1} X \mathrm{P} .\{\mathrm{X}\} \wedge \mathrm{rsem}_{1} . \mathrm{X}
\end{aligned}
$$

\subsection{Référence commune et théorie de l'esprit}

Une seconde propriété de l'interprétation du prénom, elle aussi évoquée plus haut, est le fait que (Règle 3) l'individu auquel il est fait référence est nécessairement identique pour le locuteur et l'allocutaire. Cette règle est connue de l'allocutaire,

5. La numérotation des règles est strictement pratique. Elle n'a pas de signification particulière. 
qui la sait aussi connue du locuteur. Autrement-dit, l'allocutaire s'attend à ce que le locuteur parle d'une personne que ce locuteur croit commune (« Le locuteur parle d'une personne [qui porte le prénom indiqué] qu'il pense que je connais »). L'allocutaire doit donc chercher l'individu désigné dans les connaissances TTC. C'est ce que fait la fonction srch_info2. Malheureusement, cette fonction renvoie non seulement les clefs d'individus connus, mais aussi des constantes qui ne singularisent aucun individu connu et partagé. C'est le cas de $c_{16}$ dans la section 5.3. Pour éliminer ces dernières, nous procédons comme suit. Nous savons que les individus connus et partagés sont singularisés par des clefs qui, à la différence des autres constantes individuelles, sont présentes à la fois dans les bases de connaissances TTC et CP. Pour être sûr d'avoir affaire à une telle clef, il suffit donc de s'assurer que les constantes extraites par srch_info2 apparaissent aussi dans CP. Il serait possible de recourir à une procédure logique (démonstration) mais les calculs risquent d'être fastidieux. Nous proposons plutôt de procéder à une analyse syntaxique de la base CP. Nous nous donnons un prédicat estpresent.c.bc qui renvoie vrai si le symbole c est présent dans la base bc. La règle RR1 se réécrit comme suit. Les constantes ttc et $\mathrm{cp}$ renvoient respectivement les bases de connaissances TTC et CP dans le contexte courant.

$$
\begin{aligned}
& \text { (RR1-v2) } \mathrm{GN} \leftarrow \text { prenom, } \\
& \lambda \mathrm{P} \exists_{1} \mathrm{X} P .\{\mathrm{X}\} \wedge \mathrm{X} \in \text { extraitclef.ttc.rsem } 1 \\
& \\
& \text { extraitclef }=\lambda \mathrm{BC} \lambda \mathrm{T} T \mathrm{X} \mid \mathrm{X} \in \text { srch_info2.BC.T } \wedge \text { estpresent.X.cp }\}
\end{aligned}
$$

L'application de cette interprétation au prénom dans les quatre contextes produit les résultats ci-dessous. On remarque qu'à ce stade, la représentation dit simplement qu'il existe un individu unique parmi un ensemble d'individus. L'univocité n'est donc pas encore satisfaite puisque nous attendons en plus que la représentation dise «qui» est cet individu.

contextes 1 et 2: $\lambda P \exists_{1} X P .\{X\} \wedge X \in\{$ copain, nicll $\}$

contextes 3 et 4: $\lambda P \exists_{1} X P .\{X\} \wedge X \in\{$ copain, nicll, expert $\}$

\subsection{Concurrence entre termes coréférents}

Dans le contexte 4 l'allocutaire préfère interpréter Nicolas comme l'expert, puisque «normalement» le copain est désigné par le diminutif Nico. Suivant ce même principe, il considère comme peu probable l'interprétation «tsar» car pour des individus 
ordinaires sans lien de parenté ou sans affinité particulière avec ce tsar, l'appellation usuelle est Nicolas II plutôt que Nicolas. Plus généralement, nous postulons que (Règle 4) le candidat qui ne porte pas de surnom ou diminutif dans TTC est privilégié. Si nous formulons cette règle du point de vue de l'allocutaire, nous obtenons: «Le locuteur désigne une personne qui a la double propriété: d'après lui je l'appelle par le prénom courant, mais en plus je ne l'appelle pas par un surnom. ». Si les surnoms et diminutifs sont représentés comme le prénom à l'aide d'un prédicat (sesurnommer etc.), la règle d'interprétation prend la forme ci-dessous (RR1-v3). L'idée est de lister les clefs des individus qui portent le prénom analysé et qui ne portent pas de surnom ou diminutif. Il existe de multiples façons de retranscrire cette idée. Pour comparer plus tard avec l'interprétation du nom commun, nous choisissons une formulation proche de celle adoptée par Renaud (1996) pour décrire le déterminant défini le/la. D'autres formulations sont plus adaptées pour une implémentation en vue d'un traitement automatique.

(RR1-v3) $\mathrm{N} \leftarrow$ prenom $\lambda \mathrm{P} \exists_{1} \mathrm{X}$ P. $\{\mathrm{X}\} \wedge \mathrm{X} \in$ extraitclef.ttc.rsem $1 \wedge$ restrict.ttc. $\mathrm{X}$ restrict $=\lambda B C \lambda X X \notin$ srch_info2.BC. $\left(\lambda X^{\prime} \exists E \exists Z\right.$ sesurnommer $\left.\left(E, Z, X^{\prime}\right)\right)$

restrict fait office de «prédicat de restriction». Il y a cependant une différence notoire avec celui que Renaud (1996) utilise pour désambiguïser le syntagme nominal $(\mathrm{SN})$ avec déterminant défini. Son prédicat de restriction sélectionne le candidat qui obéit aux présupposés posés par le prédicat verbal dont le SN est dépendant. Ainsi, dans Ferme la porte!, il sélectionne la porte qui est susceptible d'être fermée. Il exclu donc les portes déjà fermées. Pour cela, le prédicat de restriction doit anticiper au niveau du SN (la porte) quel sera le prédicat verbal dominant (Ferme). Bien sûr, d'un prédicat à l'autre les présupposés diffèrent. Renaud a pressenti la difficulté à concevoir un tel prédicat de restriction mais l'évacue en la reléguant au rang de simple problème technique qu'il ne résout pas. Nous estimons que l'auteur a peu de chance d'aboutir. Pour résoudre le problème, nous choisissons une toute autre stratégie. Au niveau du syntagme nominal (le prénom), nous appliquons une restriction a minima, invariante. A savoir que nous excluons les candidats usuellement appelés autrement que par leur prénom. La conformité des candidats restants avec les présupposés imposés par le prédicat verbal dominant ne sera contrôlée que plus tard dans la dérivation, une fois connu ce prédicat. Nous verrons plus loin comment se déroule le contrôle. 
Avec la version v3 de la règle d'interprétation, dans les contextes 1 à 3 , les représentations du prénom sont identiques à celles obtenues en section 6.2 . Dans le contexte 4 , la représentation vaut:

Contexte 4: $\lambda P \exists_{1} X P .\{X\} \wedge X \in\{$ nicll, expert $\}$

\subsection{Absence de toute entité de référence}

Même dans un discours collaboratif, le locuteur peut se méprendre sur les connaissances de l'allocutaire et désigner par son prénom un individu en réalité inconnu de cet allocutaire. L'allocutaire peut comprendre la méprise (« Le locuteur croit [à tort] que je connais cette personne »). Cette situation se manifeste de deux façons dans le système formel. La première est que la recherche dans TTC d'un individu portant le prénom en question renvoie un ensemble vide d'individus: l'allocutaire ne connaît personne prénommé ainsi. La seconde est que tous les candidats disponibles entraînent une incohérence de la représentation de l'énoncé. Dans les deux cas, (Règle 5) l'allocutaire peut adopter deux attitudes. Soit il interrompt le locuteur pour obtenir des informations complémentaires sur la personne désignée. C'est l'option choisie par Renaud pour le groupe nominal à base de déterminant le/la. Soit il pose l'existence d'un individu nouveau pour lui : « Soit le locuteur me parle de quelqu'un que je connais mais que l'on désigne habituellement avec un autre nom (ou surnom), soit il me parle de quelqu'un que je ne connais pas mais dont je vais désormais admettre l'existence. ». C'est la solution pour laquelle nous optons. Le choix de l'une ou l'autre solution dépend en fait de la nature de la relation entre locuteur et allocutaire. La version v4 de la règle RR1 intègre cette option.

(RR1-v4) GN $\leftarrow$ prenom

$$
\begin{aligned}
\lambda P \exists_{1} X P .\{X\} \wedge( & (\lambda T \text { si } \neg(\exists Y \text { T.Y }) \\
& \text { alors rsem } 1 . X \wedge \text { restrict.ttc. } X \\
& \text { sinon } T . X \\
) .( & \lambda Z Z Z \in \text { extraitclef.ttc.rsem } 1 \wedge \text { restrict.ttc. } Z)
\end{aligned}
$$

La représentation sémantique dit que si l'on peut démontrer que dans ttc aucun individu n'a la double propriété de se prénommer comme attendu et de ne pas porter de surnom, alors on pose l'existence d'un tel individu. Sinon, on extrait les individus concernés. 


\subsection{Interprétation du prénom et mode de l'énoncé}

Les règles évoquées dans les sections précédentes ne sont pas affectées par le mode de la phrase, que la phrase soit une négation (ex.7), une interrogative (ex.8) ou à l'impératif (ex.9). Nous postulons plus généralement que (Règle 6) les règles d'interprétation que nous énonçons dans le présent travail sont valables pour tous les modes.

...Pas de nouvelles de <Nicolas > depuis quelques temps. Tu en as? ... ... Tu as eu des nouvelles de <Nicolas $>$ ces derniers temps? ... ... Appelle vite <Nicolas $>$. Il a un truc pour toi...

\subsection{Règle de cohérence du texte}

Dans les sections précédentes, nous avons vu que la procédure d'extraction des individus portant le prénom courant ne désambiguïse pas systématiquement ce prénom. D'autres règles interviennent ultérieurement dans la dérivation de l'énoncé et contribuent à la désambiguïsation. Parmi ces règles figure une règle de cohérence (logique): (Règle 7) une interprétation incohérente est rejetée ${ }^{6}$. Il s'agit soit de la cohérence « interne » de l'énoncé soit de la cohérence par rapport au contexte. Par cohérence interne, nous entendons le fait que l'énoncé ne contient pas de contradictions, quel que soit son contexte. Par exemple tout énoncé copulatif $N$ n'est pas/ ne sont pas $N(\mathrm{ex}: * \text { Les chats }]_{N}$ ne sont pas [les chats] $\left.]_{N}\right)$ est intrinsèquement contradictoire. Comme l'énoncé étudié ici ne contient pas de contradiction interne, nous ne sommes pas concernés. Par contre, dans tous les contextes envisagés dans le présent travail, la cohérence par rapport au contexte est pertinente pour désambiguïser l'occurrence de Nicolas. Ainsi, quel que soit le contexte, l'énoncé n'interdit pas «en soi» d'attribuer à Nicolas la valeur référentielle Nicolas II. Mais cette interprétation rendrait les énoncés incohérents avec les connaissances générales selon lesquelles le personnage historique Nicolas II est mort, et que les morts ne téléphonent pas.

Dans la dérivation de l'énoncé, le contrôle de cohérence ne sélectionne pas le candidat au niveau syntaxique du prénom. Le contrôle s'effectue à des niveaux supérieurs dans l'arbre de dérivation, lorsque la représentation contient suffisamment d'éléments pour être logiquement évaluée. Dans notre exemple, nous avons besoin de disposer de l'énoncé complet pour découvrir que l'interprétation Nicolas II n'est

6. En réalité, la détection d'une incohérence n'entraîne pas systématiquement le rejet de l'énoncé. Mais pour simplifier l'exposé, nous en resterons ici à une exclusion. 
pas possible (incompatibilité avec le prédicat téléphoner). Dans d'autres énoncés, nous pourrions très bien effectuer le contrôle à un niveau plus bas dans la dérivation. Par exemple, dans le contexte 3, le syntagme nominal SN notre cher éleveur Nicolas en lieu et place de Nicolas suffirait à éliminer les candidats Nicolas II et expert-comptable, puisque aucun des deux n'est éleveur. Le contrôle de cohérence s'effectuerait alors dès l'analyse du syntagme SN, sans même disposer de l'énoncé entier. Dans tous les cas, dans la dérivation, il faut «remonter» l'ensemble des candidats jusqu'à un niveau où la représentation est évaluable. Nous verrons (section 7.1) que la grammaire utilisée réalise cette remontée.

\subsection{Règle de priorité en fonction de la présence}

Les individus sont plus ou moins «présents» dans l'environnement des locuteurs et allocutaires. Par «présence», nous entendons la présence physique d'un individu ou son évocation. Nous posons que (Règle 8) en cas d'ambiguïté, l'individu le plus présent dans «l'environnement» est la valeur référentielle privilégiée. Ainsi, dans le contexte 3, la préférence pour l'expert l'emporte car il a été le plus présent dans les dernières $24 \mathrm{H}$ de la vie du locuteur et de l'allocutaire. L'idée est déjà exploitée par les modèles cache (Kuhn \& de Mori, 1990). Avec ces modèles, le principe est de désambiguïser un mot en choisissant la valeur référentielle qu'il a le plus fréquemment reçu dans le cotexte. La définition de la notion d'environnement mériterait une étude en soi qui dépasse largement le cadre du présent article. Nous proposons une définition générale. Il s'agit d'un intervalle de temps ou d'un texte qui s'achèvent par l'énoncé courant. L'environnement peut être un événement organisé comme une réunion ou une table ronde. Le début de l'intervalle de temps est alors celui de l'événement. Une étude serait nécessaire pour proposer un chiffre raisonné dans le cas d'environnements non organisés. Nous le fixons arbitrairement à $24 \mathrm{H}$ avant la date d'énonciation de l'énoncé courant.

Pour respecter le principe de séparation des connaissances propres et attribuées, il faudrait idéalement distinguer la présence des individus pour l'allocutaire, et la théorie de l'allocutaire sur la théorie du locuteur sur l'existence des individus dans les connaissances de l'allocutaire. L'allocutaire privilégie le candidat dont il estime qu'il a été le plus présent dans cette théorie. Nous posons que dans les contextes de notre étude, la présence est la même pour le locuteur et l'allocutaire. Nous n'aurons donc pas à faire la distinction.

Nous proposons de signifier la présence d'un individu dans l'environnement de l'allocutaire par un poids associé à sa clef. Nous dotons notre modèle d'une 
base de données TTPRESENCE(env) pour chaque environnement (env) étudié. La base est constituée de couples <clef,n>. n est le nombre d'occurrences ou bien le temps de présence de l'individu singularisé par clef, dans l'environnement env. La clef n'apparaît qu'une seule fois dans la base. Dans le contexte 3 nous adoptons le temps de présence dans l'environnement constitué des dernières $24 \mathrm{H}$. Le temps de présence de l'expert durant ces dernières $24 \mathrm{H}$ étant plus élevé, son poids est plus élevé que celui du copain.

\subsection{Règle de préférence thématique}

Les individus sont plus ou moins fortement associés à des thématiques particulières. Dans les contextes étudiés ici, le copain est associé à la thématique de l'élevage tandis que l'expert est associé au monde des services, de la comptabilité. Nous posons que (Règle 9) l'interprétation privilégiée en cas d'ambiguïté et en l'absence d'autres critères de choix est l'individu dont les thématiques associées sont les plus proches des thèmes du dialogue courant. L'idée est déjà appliquée par les «topic models» (Papadimitriou et al., 1998). Considérons le dialogue dans le contexte 3. Nicolas est ambigu. Compte tenu des thématiques très spécifiques associées à chacun des Nicolas, l'ajout du titre Ex.10 (dans le cotexte de l'énoncé courant) fait basculer la préférence vers le copain. L'ajout du titre Ex.11 fait plutôt pencher la balance vers l'expert. Dans le présent travail, nous identifions chaque mot à un thème. Des conceptions plus fines sont possibles.

\section{Histoire de chèvres.}

\section{(11) Révise tes cours de comptabilité.}

Le calcul de proximité thématique nécessite de connaître d'une part les thèmes privilégiés du dialogue courant («thèmes du dialogue») et d'autre part les thèmes associés aux individus («thèmes individuels»). Lorsqu'il traite un énoncé, l'analyseur liste les thèmes privilégiés du texte, y compris dans l'énoncé courant. Si l'énoncé courant débute un dialogue, alors la liste des thèmes du dialogue est égale à la liste des thèmes de cet énoncé. Les données sont rassemblées dans une base de données BDTHEMETXT rassemblant des couples <thème, fréquence du thème>. Comme la liste comprend aussi les thèmes de l'énoncé, la liste des thèmes du dialogue évolue au fil de l'acquisition des énoncés. Par ailleurs, une base de données BDTHEMEIND contient les thèmes associés aux individus connus (dans notre cas, le copain, l'expert-comptable etc.). Cette base est constituée de triplets < clef, thème, freq $>$. freq est la fréquence des cooccurrences de l'individu (singularisé par sa clef) 
et du thème. Nous ne bornons pas a priori le nombre de thèmes pris en compte pour un individu donné. Ce nombre doit faire l'objet d'une étude. Cependant, il y a intérêt à s'en tenir aux plus fréquents. Idéalement, il faut distinguer les associations thématiques connues de l'allocutaire de celles qu'il tient pour connues du locuteur. Dans les faits, une telle finesse d'analyse est certainement difficile à obtenir. Nous n'en tenons pas compte ici. La «proximité thématique» entre un individu et le dialogue courant est la mesure de la proximité entre la liste des thèmes privilégiés du dialogue et la liste des thèmes individuels.

\subsection{Ordonnancement des règles}

Dans les sections précédentes, nous avons proposé trois règles de sélection des hypothèses d'interprétation pour un prénom ambigu. Nous supposons que ces règles coexistent et qu'elles s'appliquent dans un ordre donné. Sur la base d'une observation naïve, nous proposons de les appliquer dans l'ordre suivant (Règle 10): (1) Elimination des valeurs qui entrainent une incohérence (logique) de la représentation (voir règle 7); (2) Préférence pour la valeur la plus proche de la thématique de l'environnement (voir règle 8). (3) Préférence pour la valeur la plus fréquemment citée dans l'environnement (voir règle 9). Cet ordre est donné à titre d'hypothèse. Nous n'excluons pas qu'il puisse changer et qu'apparaissent des effets de seuils. Par exemple, la présence pourrait l'emporter lorsque son poids dépasse une valeur seuil par rapport à la valeur de la proximité thématique. Une étude à part entière serait nécessaire.

Pour chaque clef présente dans la représentation de l'énoncé courant, l'analyseur produit un «poids global» qui est un couple de poids <proximité thématique, présence>. La comparaison des poids globaux commence par la comparaison des proximités thématiques, lui donnant de facto plus d'importance. Dans le contexte 3 , le dialogue n'est marqué par aucun thème particulier. La proximité thématique ne joue donc pas. C'est la présence dans l'environnement (défini comme les dernières $24 \mathrm{H}$ ) qui décide de l'interprétation. L'expert ayant été le plus présent durant les dernières $24 \mathrm{H}$, sa présence est plus grande et son interprétation est privilégiée. C'est donc l'interprétation «expert» qui l'emporte. Si plusieurs clefs on un même plus grand poids global, alors l'analyseur procède à un tirage au sort, à défaut de disposer d'une heuristique motivée. 


\section{Modèle du processus d'interprétation du prénom}

Nous implémentons les règles vues jusqu'à présent, dans le dispositif proposé par Renaud (1996). La procédure d'interprétation des prénoms est dans ses grands traits la suivante. Au cours de la dérivation, lorsque l'analyseur rencontre un prénom, il consulte la base TTC et en extrait l'ensemble des individus qui portent ce prénom et qui sont présents aussi dans les connaissances propres. A chaque étape de la dérivation, l'analyseur tente un contrôle de cohérence. S'il peut établir qu'un des candidats entraîne une incohérence du syntagme courant, ce candidat est éliminé. Si après analyse de l'énoncé, l'ensemble contient toujours plus d'un candidat, l' analyseur (et non la grammaire) attribue un ordre de préférence aux interprétations restantes pour en choisir une (voir section 7.2).

\subsection{La grammaire}

Pour illustrer notre propos, nous présentons une petite grammaire $\mathscr{G}$ destinée à reconnaître la phrase Nicolas a téléphoné. Dans cet exemple, nous simplifions l'analyse pour nous concentrer sur la partie qui nous intéresse. Entre autres, au niveau morphosyntaxique, nous ne présentons pas les contraintes de traits. Au niveau sémantique, nous faisons abstraction des contraintes temporelles. Le verbe téléphoner est conçu ici comme un verbe intransitif.

Grammaire $\mathscr{G}$

Lexique

L1 : Nicolas, prenom, $\lambda \mathrm{X}_{l} \exists \mathrm{E}$ seprenomme $\left(\mathrm{E}\right.$, nicolas $\left._{\sigma}, \mathrm{X}\right)$

$\mathrm{L} 2$ : a téléphoné, $\mathrm{V}, \lambda \mathrm{X}_{\mathrm{ol}} \exists \mathrm{E}$ telephone $(\mathrm{E}, \mathrm{X})$

Règles

RR1-v4 : GN $\leftarrow$ prenom, $\lambda \mathrm{P} \exists_{1} \mathrm{X}_{l} \mathrm{P} .\{\mathrm{X}\} \wedge(\lambda \mathrm{T}$ si $\neg(\exists \mathrm{Y} \mathrm{T} . \mathrm{Y})$ alors (rsem1.X $X \wedge$ restrict.ttc. $X)$

sinon T.X

). $(\lambda Z Z \in$ extraitclef.ttc.rsem $1 \wedge$ restrict.ttc. $Z)$

$\mathrm{RR} 2: \mathrm{S} \leftarrow \mathrm{GN} \mathrm{V}, \mathrm{rsem}_{1} . \mathrm{rsem}_{2}$ 


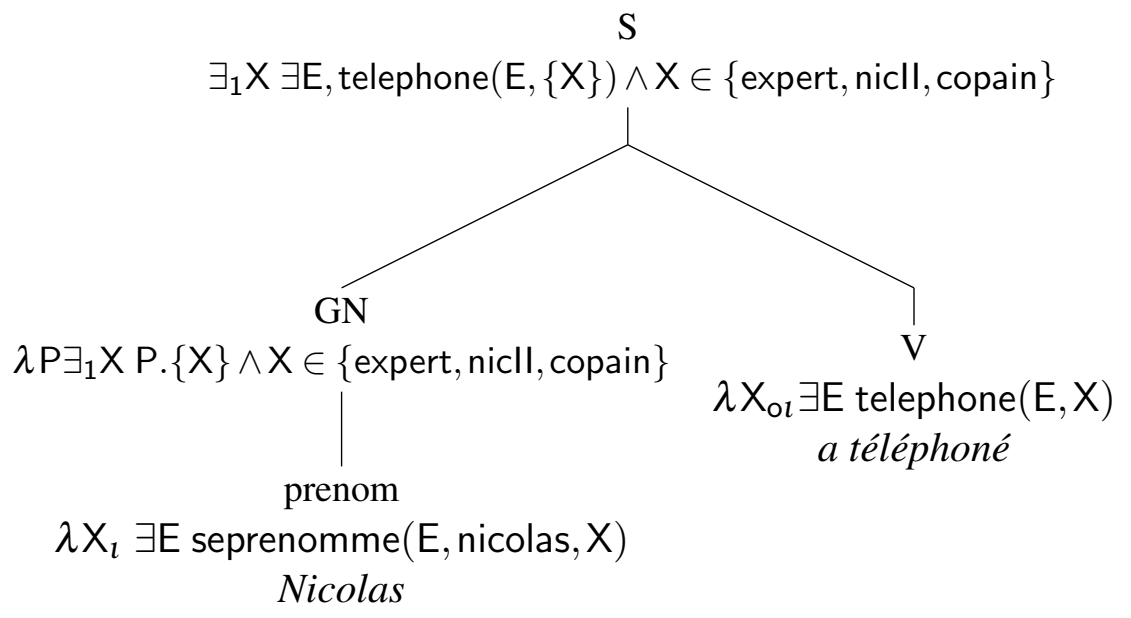

Figure 1. Dérivation de «Nicolas a téléphoné » dans le contexte 3 par la grammaire $\mathscr{G}$.

Dans les contextes étudiées, la dérivation (voir figure 1) produit les représentations suivantes, obtenues après contrôle de cohérence (pour faciliter la comparaison, les formules ne sont pas «résolues»).

Cont. 1 et $2: \exists_{1} X \exists E$ telephone $(E,\{X\}) \wedge X \in\{$ copain $\}$

Cont.3: $\exists_{1} X \exists E$ telephone $(E,\{X\}) \wedge X \in\{$ copain, expert $\}$

Cont.4: $\exists_{1} X \exists E$ telephone $(E,\{X\}) \wedge X \in\{$ expert $\}$

On constate que dans les contextes étudiés, le prénom ne reçoit pas d'interprétation « quelconque» (voir section 6.4) car dans tous les cas, au moins un candidat est disponible. Sauf dans le contexte 3 où le candidat restant n'est pas unique, l'univocité est satisfaite par la seule application de la grammaire. La désambiguïsation de l'énoncé dans le contexte 3 s'appuie sur des données non contenues dans la grammaire (voir section suivante).

\subsection{Désambiguïsation à l'aide des pondérations}

Pour le contexte 3 , rien dans la représentation sémantique ne permet de trancher entre les deux candidats restants après contrôle de cohérence. Pour désambiguïser, l'analyseur exploite les données extérieures à la grammaire: proximité thématique et présence. 


\section{Raoul Blin}

L'heuristique consiste premièrement à attribuer un «poids» à chaque interprétation possible en fonction du poids des clefs. Puis deuxièmement l'interprétation de poids le plus élevé est retenue. Si une interprétation $T_{0}$ contient un sous-terme ST de la forme $\exists_{1} X \ldots X \in\left\{c_{1}, \ldots, c_{n}\right\}$, on produit l'ensemble des termes $T_{i \in[1, n]}$ qui valent $T_{0}$ dans lequel $X=c_{i}$ est substitué à $X \in\left\{c_{1}, \ldots, c_{n}\right\}$. Cette opération est appliquée de façon récursive jusqu'à ce que les termes ainsi dérivés de $T_{0}$ ne contiennent plus de sous-terme ST. Cette procédure appliquée à la représentation sémantique obtenue dans le contexte 3 (voir section précédente) produit l'ensemble de termes suivant :

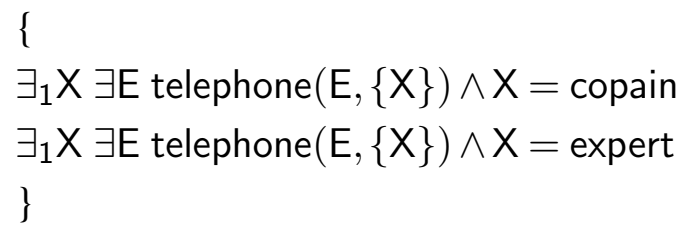

Les termes qui contiennent une contradiction sont éliminés (en l'occurrence, aucun n'est concerné ici). A chaque terme restant, nous attribuons un «poids» calculé en sommant les poids des clefs qu'il contient (d'autres méthodes de calcul peuvent être utilisées). Dans notre exemple, chaque terme ne contenant qu'une seule clef, son poids vaudra celui de la clef. Nous savons que dans le contexte 3 , c'est la clef de l'expert qui est la plus grande. Pour finir nous sélectionnons le terme de poids le plus élevé. La représentation sémantique de l'énoncé vaut au final: $\exists_{1} X \exists E$ telephone $(E,\{X\}) \wedge X \in\{$ expert $\}$, c'est-à-dire: $\exists E$ telephone $(E,\{$ expert $\})$. $\mathrm{Au}$ cas où les poids seraient identiques, nous suggérons de recourrir par défaut à un tirage au sort, en attendant une solution motivée.

\section{Conclusion}

Nous avons proposé un modèle du processus de désambiguïsation du prénom en contexte, et montré comment articuler des données hétérogènes, comme par exemple la théorie de l'esprit, la cohérence logique des énoncés, ou encore la préférence thématique. Nous avons par ailleurs montré qu'il était possible de s'en tenir à une logique classique monotone (théorie des types). Pour la discussion, nous avons volontairement limité le champ d'observation. Nous nous en sommes tenu à l'observation du prénom dans son usage «pur», dans un nombre limité de contextes, et, surtout, dans des énoncés sans cotexte. Cela signifie que l'individu porteur du prénom n'apparaît pas dans le cotexte immédiat. Une prochaine étape de la recherche consistera à observer dans quelles conditions un prénom peut être utilisé pour désigner une personne qui vient d'être évoquée dans le cotexte immédiat. Si un tel usage 
du prénom est possible, il faut étudier l'impact sur le processus de désambiguïsation. En particulier, un candidat qui vient juste d'être évoqué est-il l'interprétation privilégiée, voire même la seule interprétation possible? Dans les deux cas, cela signifie que le critère de proximité dans le discours l'emportera sur les autres critères (présence et proximité thématique).

Nous faisons l'hypothèse que de nombreux éléments de la procédure de désambiguïsation, voire la procédure entière, sont applicables à d'autres structures et mots. Nous pouvons tout d'abord améliorer la représentation du déterminant défini le/la de Renaud (1996) en résolvant le problème de la définition du prédicat de restriction. Comme pour le prénom, et contrairement à l'auteur, nous ne cherchons pas à résoudre l'ambiguïté au niveau du syntagme du prénom seul. Les ambiguïtés sont résolues plus tard dans la dérivation. Pour le déterminant défini, le prédicat de restriction n'est alors plus nécessaire. Nous reformulons la représentation du déterminant défini comme suit, en nous inspirant de celle du prénom.

$$
\begin{aligned}
\text { le, Det, } \lambda N \lambda P \exists_{1} X P .\{X\} \wedge(\lambda T \text { si } \neg(\exists Y T . Y) \\
\text { alors } N . X \\
\text { sinon } T . X \\
) .(\lambda Z Z \in \text { extraitclef.ttc. } N)
\end{aligned}
$$

Une observation naïve laisse penser que la procédure de désambiguïsation proposée dans cet article vaut aussi pour de nombreux autres noms propres nus. C'est le cas des toponymes (ex. Paris), des patronymes de toutes sortes comme les noms de famille Durand a téléphoné., les surnoms etc. Enfin considérant les noms propres de la forme <déterminant + nom propre nu> (ex. la Seine, l'Elysée) comme des formes figées, nous estimons que notre procédure assure aussi leur traitement. La différence majeure est certainement que le nombre de candidats est bien plus limité que pour le prénom.

Pour décrire l'usage de la théorie de l'esprit dans le processus de désambiguïsation, nous avons structuré les connaissances générales en trois sous-ensembles (CP connaissances propres, TC théorie sur les connaissances et TTC théorie sur les théories sur les connaissances). Nous avons vu comment utiliser ces sous-ensembles en interprétation. Mais cette structure est aussi utilisable en génération, notamment pour choisir les verbes cognitifs (savoir, croire, penser, etc.), et sans recourir à des logiques modales et/ou non monotones. Le choix du verbe se ferait tout simplement en fonction de la localisation de l'information à transmettre. Un examen exhaustif reste à faire. Voici quelques exemples. 
(1) a. Connaissances propres, stockées dans CP : (Je <sais> que) Nicolas a téléphoné.

b. Information stockée dans la Théorie sur les connaissances de Pierre (TC) et dans les connaissances propres du locuteur (CP): Pierre < sait $>$ que Nicolas a téléphoné [et je le sais aussi].

c. Connaissance attribuée à Pierre, mais sur laquelle le locuteur ne peut se prononcer. Autrement-dit, l'information est présente dans TC mais pas dans CP. Sa contraposée ne figure pas non plus dans CP: Pierre <pense> que Nicolas a téléphoné. .

d. Fausse connaissance attribuée à Pierre. L'information figure dans TC et sa contraposée figure dans CP: Pierre < croit> que Nicolas a téléphoné..

\section{Euvres citées}

Andrews P. B. (1986). An introduction to mathematical logic and type theory: to truth through proof. Computer science and applied mathematics. Orlando: Academic Press.

Blin R. (2009). Introduction à la linguistique formelle. TIC et sciences cognitives. Paris: Hermès science publications : Lavoisier.

Bratman M. (1999). Intention, plans, and practical reason. David Hume series. Stanford, Calif: Center for the Study of Language and Information.

Davidson D. (2001). Essays on actions and events. Oxford : New York, 2nd ed edition. 04155.

Dennett D. C. (1978). Beliefs about beliefs. Behavioral and Brain Sciences, 1, 568-570.

Enjalbert P. (1996). De l'interprétation (sens, structures et processus). Intellectica, 23, 79-120.

Fredge G. (1971). Ecrits logiques et philosophiques. Paris.

Gosselin L. (1996). Le traitement de la polysémie contextuelle dans le calcul sémantique. Intellectica, 1(22), 93-117.

Jonasson K. (1994). Le nom propre: constructions et interprétations. Champs linguistiques. Louvain-la-Neuve: Duculot.

Kamp H. \& Reyle U. (1993). From Discourse to Logic: Introduction to Modeltheoretic Semantics of Natural Language, Formal Logic and Discourse Representation Theory. Number 42 in Studies in Linguistics and Philosophy. Kluwer Academic Publishers.

Kleiber G. (1981). Problème de référence : description définie et nom propre. Number 6 in Recherches linguistiques. [Metz] : Paris: Centre d'analyse syntaxique de l'Université de Metz; Klincksieck [distributor].

Kripke S. (1972). Naming and Necessity. In Semantics of Natural Language, p. 181-201. Davidson, D. et G.Harman, dordrecht edition.

Kuhn R. \& de Mori R. (1990). A cache-base natural language model for speech recognition. In IEEE PAMI, volume 12, p. 570-583.

Lyons J. (1978). Semantics. Paris.

Norimatsu H., Blin R., Hashiya K., Sorsana C. \& Kobayashi H. (2014). Understanding of others' knowledge in French and Japanese children: a comparative study with a disambiguation task on 16-38-month-olds. Infant Behavior \& Development, 37(4), 632-643.

Papadimitriou C. H., Tamaki H., Raghavan P. \& Vempala S. (1998). Latent semantic indexing: a probabilistic analysis. p. 159-168: ACM Press.

Premack D. \& Woodruff G. (1978). Does the chimpanzee have a theory of mind? Behavioral and Brain Sciences, 1(04), 515. 
Pynadath D. V. \& Marsella S. C. (2005). Psychsim: Modeling theory of mind with decision-theoretic agents. In IJCAI, volume 5, p. 1181-1186.

Renaud F. (1996). The definite article: code and context. Journal of Semantics, 13(2), 139-180.

Renaud F. (2005). Temps, durativité, télicité. Number 60 in Bibliothèque de l'Information Grammaticale. Louvain, Paris: Peeters.

Sabouret n. \& Belkaid M. (2014). Un modèle logique de théorie de l'esprit pour un agent virtuel dans le contexte de simulation d'entretien d'embauche. In Proc. Workshop Affect, Compagnon Artificiel, Interaction, Rouen.

Searle J. (1971). The problem of proper names. p. pp.135-141. Steinberg, d. \& jakobovits, 1. edition.

Wimmer H. \& Perner J. (1983). Beliefs about beliefs: Representation and constraining function of wrong beliefs in young children's understanding of deception. Cognition, 13(1), 103-128.

\section{Résumé}

Un nom propre, parfois porté par des centaines de milliers d'individus, lorsqu'il apparaît sans déterminant dans un énoncé, ne peut faire référence qu'à un seul et unique individu, non quelconque et identique pour le locuteur et son allocutaire. Une bonne part de la désambiguïsation repose sur l'usage des données contextuelles. Nous proposons un modèle opératoire du processus d'interprétation du nom propre, et plus précisément du prénom. Nous mettons en particulier l'accent sur l'articulation entre la grammaire et les connaissances générales, ainsi que la théorie de l'esprit.

\section{Summary}

A few thousand individuals can have the same first name but in a sentence, and without articles, a first name can refer to one person only and this person must be known to both the locutor and interlocutor. Moreover, the referential value is the same for both. Disambiguation is strongly dependent on contextual knowledge. In this paper we propose a functional model of the process by which individuals disambiguate the referential value of proper nouns, in particular first names. We focus on the interplay between grammar, general knowledge and the theory of mind.

\section{Mots-clés}

Résolution d'ambiguïté, nom propre, théorie de l'esprit, modèle formel. 
28 Raoul Blin

\section{Keywords}

Ambiguity resolution, proper noun, theory of mind, formal model.

Adresse de l'auteur :

CRLAO-EHESS, 105 Boulevard Raspail, 75006 Paris 